\title{
Radiographic patterns and response to corticosteroid hip injection
}

\author{
M J Plant, A A Borg, K Dziedzic, J Saklatvala, P T Dawes
}

Department of Rheumatology, Wrexham Maelor Hospital, Wrexham M J Plant

Department of Rheumatology, Nevill Hall Hospital, Abergavenny A A Borg

Staffordshire Rheumatology Centre, Haywood Hospital, Stoke on Trent K Dziedzic P T Dawes

Department of Radiology, North Staffordshire Hospital Trust,

Stoke on Trent

J Saklatvala

Correspondence to: Dr M J Plant, Department of Rheumatology, Wrexham Maelor Hospital, Croesnewydd Road, Wrexham, Clwyd LL13 7TD.

Accepted for publication 6 June 1997

\begin{abstract}
Objectives-A prospective, open study of corticosteroid hip injection (CHI) was performed to determine if different radiological patterns of arthritis vary in their response.
\end{abstract}

Methods-Forty five patients (15 with rheumatoid arthritis, 27 with osteoarthritis, and three with anklyosing spondylitis) underwent hip injection with $80 \mathrm{mg}$ methylprednisolone and lignocaine under $x$ ray control. Outcome was assessed at two, 12, and 26 weeks for pain, range of hip movement, and graded functional questionnaire. Patients estimated their pain in four components, night pain, rest pain, weight bearing, and referred pain, each measured by $10 \mathrm{~cm}$ visual analogue score and summed to give a total score out of 40 cm. Hip radiographs were evaluated blindly for pattern and severity of arthritis, as well as for progression between 0 and 26 weeks.

Results-Median total pain score decreased from $29 \mathrm{~cm}$ at baseline to $22 \mathrm{~cm}$ at two weeks $(p=0.0001), 24 \mathrm{~cm}$ at 12 weeks $(p=0.03)$, but had returned nearly to baseline by 26 weeks $(25 \mathrm{~cm}, p=0.3)$. Greatest improvement was seen for night pain. Mean range of internal rotation increased from 16 to 28 degrees at two weeks $(p=0.03)$ and 23 degrees at 12 weeks $(p=0.06)$. Functional ability did not change. Hips with an atrophic pattern of arthritis on plain radiography gained negligible pain relief at two weeks compared with hips with a hypertrophic or mixed bone response $(p=0.04)$. The degree of pain relief was similar in patients with $O A$ and $R A$, and was not influenced by radiographic severity or by the direction of migration of the femoral head.

Conclusion-Pain and internal rotation improve for up to 12 weeks after CHI. CHI offers a useful and safe therapeutic option for patients with hip arthritis, with the exception of those with a purely atrophic radiological pattern.

\section{(Ann Rheum Dis 1997;56:476-480)}

Arthritis of the hip is a significant cause of morbidity in the elderly population. Osteoarthritis (OA) is the commonest cause and affects $4 \%$ of people over the age of 65 years. ${ }^{1}$ The prevalence of hip involvement in rheumatoid arthritis (RA) may approach $50 \%$ in established disease ${ }^{2}$ with protrusio acetabuli in $5 \%{ }^{3}$ Although ankylosing spondylitis (AS) is uncommon, the hip is the most commonly involved of the peripheral joints. Total hip replacement provides a successful intervention in end stage hip arthritis, but it is often preceded by years of pain and disability. Moreover, elderly patients may not be suitable for surgery or may not be referred. ${ }^{4}$ Intra-articular corticosteroids are widely used in inflammatory joint diseases, although their use in OA is more controversial, partly because of lesser efficacy and also because of concerns over deleterious effects. ${ }^{56}$

Recently hip arthritis has been separated into radiological subsets. ${ }^{6}$ The pattern of bone response has been separated into atrophic and hypertrophic types, both of which occur in OA and RA. Atrophic hip arthritis is associated with chondrocalcinosis at the hip and tends to progress more rapidly. ${ }^{7-9}$ Using the direction of migration of the femoral head, OA has been divided into superior pole and medial (central) types. Superior migration is more common in men, more often unilateral, and more likely to progress. ${ }^{810}$ Medial OA is more common in women, tends to be bilateral, and is less likely to progress $^{810}$ : there are conflicting data regarding its association with generalised nodal OA. ${ }^{71}$ In OA $82 \%$ of hips show superior pole migration, ${ }^{7}$ whereas in RA axial migration is the most common pattern. If these subsets do represent distinct pathological categories, they may respond differently to corticosteroid injection; this question has not previously been studied.

The objectives of this prospective, open study were: (1) to evaluate the changes in pain, function, and range of movement after corticosteroid hip injection (CHI) under radiological guidance; (2) to determine which radiographic types of hip disease respond best.

\section{Methods}

PATIENTS

Forty five adult patients underwent CHI over a two year period. Eleven patients were recruited from the orthopaedic waiting list for hip replacement and 34 were referred from routine rheumatology clinics. Criteria for inclusion were: (1) hip pain for more than one month requiring regular analgesia, (2) pain on weight bearing and at night, (3) restriction of hip movement. Exclusion criteria comprised: (1) pregnancy, (2) Paget's disease of hip or pelvis, (3) previous surgery or corticosteroid injection to the affected hip, (4) suspected sepsis of hip or surrounding tissues. 
HIP INJECTION

All patients received an injection of $4 \mathrm{ml} \mathrm{1 \%}$ lignocaine and $80 \mathrm{mg}$ methylprednisolone (Depomedrone, Upjohn) into the synovial cavity of the affected hip. A $22 \mathrm{G}$ spinal needle was introduced under fluoroscopy by the anterior approach, and intra-articular position was confirmed by instillation of $2 \mathrm{ml}$ of contrast medium (Ultravist 300) in all patients. All injections were performed by the same operator (AAB). Patients were returned to the ward by chair for four hours bed rest, before discharge in the early evening. They were not given any special instuctions thereafter and were allowed to resume usual activity. Local ethical committee approval was obtained before commencement of the study.

ASSESSMENT OF OUTCOME

Patients were assessed by the same physiotherapist for pain, functional ability, and range of movement (ROM). Assessments were made three hours before the injection (baseline) and after two, 12, and 26 weeks.

Pain was assessed by four $10 \mathrm{~cm}$ visual analogue scales (VAS) with reference to night pain, rest pain, weight bearing pain, and referred pain. These were summed to give a total pain score $(\max 40 \mathrm{~cm})$. Pain relief at two weeks was calculated by subtracting the total pain score at two weeks from the baseline score.

Movement was measured by a goniometer in both hips for flexion, abduction, internal rotation, external rotation and extension; leg length was also determined.

Functional ability was estimated using a lower limb questionnaire ( $0-13$ scale), graded according to walking distance. $1=$ walk more than five miles. $3=$ walk more than one mile. 5 $=$ walk $>100$ yards. $7=$ walk $<100$ yards (if walking stick required, add 1 mark.). $9=$ walk upstairs. $11=$ rise from chair. $13=$ chairbound.

\section{RADIOGRAPHY}

Anteroposterior radiographs of the hip were taken at baseline and at 26 weeks. These radiographs were graded by one observer (MJP) with training in musculoskeletal radiology, and who was unaware of the primary diagnosis, clinical outcome or physiotherapy measurements. The joint space was measured in millimetres at the narrowest point. Overall grading of severity was made according to the scales of Kellgren and Lawrence ${ }^{12}$ for OA, and by Larsen's method for RA. ${ }^{13}$ The pattern of bone response was described as being atrophic or hypertrophic or mixed. ${ }^{14}$ Cysts were estimated on a $0-3$ scale in both the femoral head and the acetabulum $^{15}$ : $0=$ none, $1=$ one or two small cysts, $2=$ single large or multiple small, $3=$ multiple large cysts. Other features graded on a 0 to 3 scale included joint space narrowing (JSN), osteophyte formation, and sclerosis. ${ }^{16}$

The pattern of migration of the femoral head was defined as superior, medial, axial or indeterminate.$^{11}$ For analysis, medial and axial groups were combined and compared with the superior group; four radiographs in which the migration pattern could not be determined were excluded from the analysis.

Follow up $x$ rays after six months were available on 39 patients. The paired films were blinded to order by covering the patient identification area and serial films were compared for radiological change.

\section{STATISTICS}

The study was analysed on an intention to treat' basis: for patients who withdrew before 26 weeks, their last measures were carried forward. Analyses were performed using the NCSS statistical software package (Number Cruncher Statistical System, version 5.01: Dr J L Hintze, Kaysville, Utah). Pain and function data were analysed by non-parametric methods, using Wilcoxon signed rank test and the Kruskal-Wallis test: measurements of range of movement were compared by paired $t$ test. Reproducibility of grading of radiographs was tested by $\kappa$ statistic, which compares the observed proportional agreement with the agreement that would have been expected by chance.

\section{Results}

PATIENT CHARACTERISTICS

Forty five patients ( 10 male: 35 female) entered the study The underlying disease was RA in $15, \mathrm{OA}$ in 27, and AS in three. Mean age was 59 years, range 21-79. A wide range of radiological abnormality was covered: minimum joint space width ranged from $0 \mathrm{~mm}$ to 5 $\mathrm{mm}$ (median $1 \mathrm{~mm}$ ), Kellgren OA grade from 1 to 4 (median 3), and Larsen grade from 1 to 5 (median 3).

All 45 patients were assessed at baseline and at two weeks; 37 patients were reviewed at three months, and 30 at six months. Outcome was compared at the four time points using intention to treat analysis: for the 15 patients who withdrew early their last measurements were carried forward. Factors affecting response to CHI were investigated in the complete study group at the two week assessment.

Of the 15 study withdrawals, nine were lost because of hip surgery and six declined or failed to attend for follow up. The withdrawal group had higher baseline pain scores (29.8 $v$ 26.3), but this was not statistically significant $(p=0.22)$. The groups were similar for age, initial function, and radiographic severity. Hip movement tended to be more restricted in the withdrawal group, but this was only significant for flexion $\left(61^{\circ} v 78^{\circ} ; \mathrm{p}=0.01\right)$, and internal rotation was actually slightly greater in the withdrawal group $\left(21^{\circ} v 16^{\circ}\right)$. Median pain relief at two weeks was the same for study drop outs and completers (6.1 $v 6.2)$, and so the patients who defaulted were not all non-responders.

\section{OUTCOME}

Median total pain score $(\max =40 \mathrm{~cm})$ decreased from $28.5 \mathrm{~cm}$ at baseline to $22.4 \mathrm{~cm}$ at two weeks, $23.8 \mathrm{~cm}$ at 12 weeks and had returned to near pre-injection values $(24.9 \mathrm{~cm})$ by 26 weeks (fig 1 and table 1). The improvement with respect to baseline was highly 


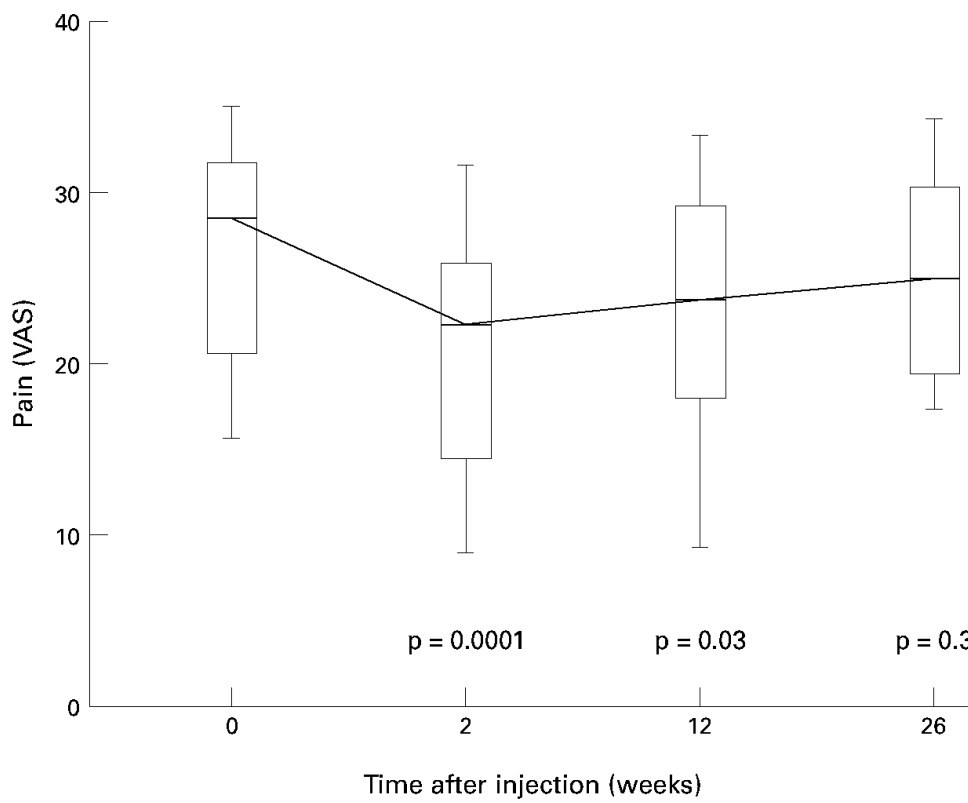

Figure 1 Total hip pain after corticosteroid hip injection. Total hip pain by visual analogue scale ( $\max =40 \mathrm{~cm}$ ) is shown in 30 patients followed up for six months after injection of $80 \mathrm{mg}$ methylprednisolone under $x$ ray guidance. The middle bar represents the median: the box represents the $25 \%$ and $75 \%$ quartiles: the whiskers represent the $10 \%$ and $90 \%$ centiles. $p$ Values are for comparison with baseline pain scores (Wilcoxon signed rank test).

Table 1 Pain and functional ability after corticosteroid hip injection

\begin{tabular}{lllll}
\hline \multicolumn{5}{l}{ Time after corticosteroid hip injection } \\
\cline { 2 - 5 } & O weeks & 2 weeks & 12 weeks & 26 weeks \\
\hline Total pain & $28.5(21-32)$ & $22.4^{\star \star \star}(14-26)$ & $23.8^{\star}(18-29)$ & $24.9(19-30)$ \\
Night pain & $7.5(5-9)$ & $4.9^{\star \star \star}(2-7)$ & $6.6^{\star}(3-8)$ & $6.7(4-8)$ \\
Rest pain & $5.4(3-8)$ & $3.3^{\star \star}(2-5)$ & $4.0(3-7)$ & $4.7(3-7)$ \\
Weight bearing pain & $8.6(7-9)$ & $6.6^{\star \star \star}(4-9)$ & $7.3^{\star}(5-9)$ & $7.5(6-9)$ \\
Referred pain & $7.3(5-8)$ & $5.4^{\star \star}(3-8)$ & $5.9(4-8)$ & $6.9(5.3-7.7)$ \\
Function range 0-13 & $5(4-7)$ & $6(4-7)$ & $5(4-7)$ & $5.5(5-7)$
\end{tabular}

The values are medians (interquartile range) for pain by $10 \mathrm{~cm}$ VAS; total pain is the sum of the components of night, rest, weight bearing, and referred pain (maximum $=40 \mathrm{~cm})$. Significan change from baseline is shown; ${ }^{\star} \mathrm{p}<0.05,{ }^{\star \star} \mathrm{p}<0.01,{ }^{\star \star \star} \mathrm{p}<0.001$ (Wilcoxon signed rank test).

Table 2 Pain relief after corticosteroid hip injection related to underlying disease and radiographic features

\begin{tabular}{|c|c|c|c|c|}
\hline & & $\begin{array}{l}\text { Pain relief at } 2 \\
\text { weeks median }\end{array}$ & $\begin{array}{l}\text { Pain relief at } 2 \text { weeks } \\
\text { confidence limits }\end{array}$ & p Value \\
\hline \multicolumn{5}{|l|}{ Diagnosis } \\
\hline $\mathrm{RA}$ & $\mathrm{n}=15$ & 6.2 & -4.4 to 13.5 & \multirow[t]{3}{*}{$\mathrm{p}=0.9 \mathrm{NS}$} \\
\hline $\mathrm{OA}$ & $\mathrm{n}=27$ & 6.6 & 1.3 to 11.8 & \\
\hline AS & $\mathrm{n}=3$ & 5.7 & NA & \\
\hline \multicolumn{5}{|c|}{ Pattern of bone response } \\
\hline Atrophic & $\mathrm{n}=13$ & 0.9 & -3.3 to 6.7 & \multirow[t]{3}{*}{$\mathrm{p}=0.04^{\star}$} \\
\hline Mixed & $\mathrm{n}=18$ & 7.5 & 4.8 to 14.3 & \\
\hline Hypertrophic & $\mathrm{n}=14$ & 7.6 & 3.4 to 12.0 & \\
\hline \multicolumn{5}{|c|}{ Radiographic severity (minimum joint space width) } \\
\hline $0-1 \mathrm{~mm}$ & $\mathrm{n}=24$ & 6.2 & -2.9 to 8.2 & \multirow{3}{*}{$\mathrm{p}=0.77 \mathrm{NS}$} \\
\hline $2-3 \mathrm{~mm}$ & $\mathrm{n}=9$ & 6.0 & 0.2 to 14.3 & \\
\hline $4-5 \mathrm{~mm}$ & $\mathrm{n}=12$ & 10.3 & -9.3 to 25.7 & \\
\hline \multicolumn{5}{|c|}{ Direction of migration of femoral head } \\
\hline Superior & $\mathrm{n}=22$ & 6.9 & 5.1 to 12.0 & \multirow[t]{2}{*}{$\mathrm{p}=0.37 \mathrm{NS}$} \\
\hline Medial/axial & $\mathrm{n}=19$ & 3.3 & -3.3 to 11.3 & \\
\hline
\end{tabular}

The values are median relief of total pain at 2 weeks (confidence limits of median): a positive figure indicates reduction of pain, and a negative figure indicates an increase in pain relative to baseline. Data were analysed for differences between groups by Kruskal-Wallis or

Mann-Whitney tests for non-parametric groups (NS = not significant).

significant at two weeks $(\mathrm{p}=0.0001)$, and still significant at 12 weeks $(\mathrm{p}=0.03)$. Night pain, rest pain, referred pain, and weight bearing pain all improved, particularly night pain (table 1). These findings were not changed by restricting the analysis to the 30 patients who completed all study visits.
Nineteen patients achieved at least a $25 \%$ decrease in total pain score at two weeks; of this group 12 patients maintained $25 \%$ improvement to 12 weeks and five to 26 weeks. Median pain relief at two weeks in all 45 patients was similar for OA, RA, and AS patients (table 2).

Functional ability showed no change (table 1). Internal rotation increased significantly from a mean of 17 degrees to 27 degrees at two weeks, but had fallen back to 23 degrees by 12 weeks $(p=0.06)$ (table 3$)$. Other movements showed no change; there were no significant changes in ROM for the non-injected hip.

\section{RADIOGRAPHY}

To determine if the radiological pattern of hip disease influenced the response to injection, the atrophic, hypertrophic, and mixed subtypes were compared in terms of the pain relief obtained at two weeks - total pain at 0 weeks minus pain at two weeks (table 2). Pain relief was significantly less in atrophic hips (median $0.9 \mathrm{~cm}$ ) compared with mixed or hypertrophic ones (median $7.5 \mathrm{~cm}$ and $7.6 \mathrm{~cm}$ respectively). One possible statistical flaw of this analysis is that if atrophic hips tended to have less initial pain, they would be less able to demonstrate pronounced pain relief (regression towards the mean). However, the mean initial pain for atrophic hips was $26 \mathrm{~cm}$ compared with $29 \mathrm{~cm}$ for hypertrophic/mixed hips ( $p>0.6)$, making this unlikely to have an important impact.

The degree of radiological severity as judged by minimum joint space had no effect on pain relief after CHI (table 2); neither was there any significant difference in pain relief when analysis was made by Kellgren grade for the OA patient group $(p=0.6)$ or by Larsen grade for the RA patients $(p=0.2)$. The direction of migration of the femoral head did not seem to influence pain relief and nor did the degree of joint space narrowing, cysts or osteophyte formation.

Fifteen of 39 hips (38\%) deteriorated radiographically at 26 weeks compared with baseline, although only five actually increased their Larsen or Kellgren grade. The hips that showed radiological worsening tended to have more severe initial disease; they had a narrower joint space (mean of $0.8 \mathrm{~mm} v 2.1 \mathrm{~mm}$, $\mathrm{p}=0.01)$ and a worse overall grade (3.3 $v 2.5$ for OA hips by Kellgren and $3.4 v 2.4$ for Larsen RA hips, $p=0.006)$. There was a tendency for atrophic hips and those with a superior migration pattern to progress more often, but this was not significant using the $\chi^{2}$ test with Yates's correction ( $p=0.4$ and $p=0.11$ respectively).

REPRODUCIBILITY OF RADIOGRAPHIC SCORING Intraobserver reproducibility was tested on a subset of 19 hip radiographs that were still available to be rescored by the primary observer after an interval of 12 months. The observed proportional agreement $\left(\mathrm{p}_{\mathrm{o}}\right)$ for the various radiographic features was: JSN $79 \%$ $(\kappa=0.71)$, osteophyte $68 \%(\kappa=0.53)$, sclerosis $47 \%(\kappa=0.11)$, cysts $58 \%(\kappa=0.45)$, Kellgren grading $68 \%(\kappa=0.53)$, Larsen grading $47 \%$ $(\kappa=0.28)$, direction of migration of femoral 
Table 3 Range of movement after corticosteroid hip injection

\begin{tabular}{lllll}
\hline & \multicolumn{4}{l}{ ROM after corticosteroid hip injection mean degrees (SD) } \\
\cline { 2 - 5 } & 0 weeks & 2 weeks & 12 weeks & 26 weeks \\
\hline Internal rotation & $17(14)$ & $27(20)^{\star \star}$ & $23(18)$ & $22(18)$ \\
External rotation & $43(18)$ & $43(18)$ & $42(18)$ & $45(18)$ \\
Flexion & $78(21)$ & $82(25)$ & $78(25)$ & $77(19)$ \\
Abduction & $24(11)$ & $25(10)$ & $24(10)$ & $24(10)$ \\
\hline
\end{tabular}

The values are means (SD) in degrees. There was no change in extension or leg length; ROM for the non-injected hip did not change significantly (data not shown). ${ }^{\star \star}=\mathrm{p}<0.01$

(paired $t$ test). because patients were told that they would be given priority for surgery if their pain worsened after injection. An earlier paper in 1956 showed that hips treated with hydrocortisone and lidocaine do gain greater subjective improvement than with lidocaine alone. ${ }^{19}$ In arecent retrospective analysis of 38 patients injected with hydrocortisone and lignocaine, hip pain improved after $69 \%$ of procedures for more than three months. ${ }^{20}$ However, these results should be interpreted with caution, as a major element of recall bias cannot be excluded.

Recent data have suggested that immobilisation for 24 hours after injection of weight bearing joints confers a more longlasting effect. ${ }^{21}$ In our study patients were rested in bed for four hours; it is possible that a longer period of bed rest might further improve the efficacy.

A control group treated by placebo injection was not included in this study as it was felt to be unethical because the patients all had severe symptoms not responding to maximal analgesia. We would accept that part of the improvement shown could have been a placebo effect, and so the results should be interpreted with caution while awaiting randomised comparative studies. However, the primary objective of this study was to discover if the radiographic pattern of arthritis had any effect upon response to $\mathrm{CHI}$. Indeed, the pattern of bone response was found to be an important factor in response to injection and it proved a reliable measure both within and between observers. Atrophic hips did not gain any significant benefit compared with hips with a hypertrophic or a mixed bone response. Consequently, patients with atrophic disease should perhaps be considered for arthroplastic surgery rather than for corticosteroid injection. This interesting finding may be explained by either atrophic disease being less inflammatory and hence less corticosteroid responsive, or alternatively corticosteroid may actually worsen bone resorption in these hips-this has been reported as a possible adverse effect of such injections. ${ }^{22}$

The radiographic severity of the hip arthritis did not have an important influence on response to CHI. Severity was graded by minimum joint space as well as by Kellgren and Larsen scales as the former proved to be a more reproducible measure. It is also noteworthy that patients with RA and OA responded equally well. In contrast with our results, other workers have found greater improvement in RA than OA, both in the hip ${ }^{20}$ and the knee. ${ }^{22}$ This might be explained by the fact that, in this study, atrophic disease was more common in RA patients (six of 15) compared with OA patients (six of 27).

Predictive factors for response to intraarticular corticosteroid have not previously been investigated for the hip joint. Two recent studies have attempted to identify such factors in the osteoarthritic knee. ${ }^{232}$ Gaffney et al found increased benefit in patients with evidence of joint effusion, but radiographic severity did not seem to be important. ${ }^{23}$ Conversely, Jones et al were unable to identify any clinical predictors, although radiographic triamconolone. Between $58 \%$ and $75 \%$ patients improved in all groups. However, this small trial was biased towards a negative result 
parameters were not investigated. In both studies improvement over placebo was seen in the short-term (1-3 weeks) but not over the medium term (6-8 weeks).

In this study, 15 of $39(38 \%)$ of hips showed radiological worsening after six months. Although this rate may seem high it should be remembered that serial films were compared for any small degree of change and the percentage showing a discrete change in Kellgren or Larsen grade was less $(12 \%)$. The hips showing radiographic progression had significantly more severe initial disease with more subchondral cysts and greater JSN. Atrophic and superior migration pattern hips tended to progress more often but this was not statistically significant because of the small numbers in each group; however, these findings are in keeping with other studies. ${ }^{8}$

In our experience accurate intra-articular hip injection requires $x$ ray screening as surface landmarks are unreliable. The procedure was well tolerated by the patients and the technique was easily learnt by the operator; up to three patients could be treated in one hour of $x$ ray time. Radiological exposure is minimal with an average screening time of 30-60 seconds. There are several clinical situations in which CHI may be particularly useful: (1) when surgery is contraindicated because of coexisting medical conditions, (2) in young patients when there is concern over the potential longevity of a hip prosthesis, (3) as a diagnostic trial to confirm the hip as the source of pain, (4) patients awaiting hip replacement who might benefit not only from temporary amelioration of symptoms but also reduction in the need for potentially toxic non-steroidal antiinflammatory treatment.

In conclusion, this open study suggests that $\mathrm{CHI}$ can improve pain and internal rotation for up to 12 weeks in both inflammatory arthritis and osteoarthritis. However, response is negligible in atrophic hips compared with those with a hypertrophic or mixed bone response. We feel that CHI offers a further therapeutic option for hip arthritis in those patients whose radiological pattern is not purely atrophic.

We should like to thank Dr T E Hothersall and Dr M F Shadforth for referring their patients, and the Haywood Foundation, which funded Dr A A Borg and Ms K Dziedzic. Upjohn Pharmaceuticals Ltd kindly supplied Depomedrone.
1 Lawrence JS, Bremner JM, Bier F. Osteoarthrosis: Prevalence in the population and relationship between symptoms and x-ray changes. Ann Rheum Dis 1966;25:1-24.

2 Duthie R, Harris C. A radiographic and clinical survey of the hip joints in seropositive rheumatoid arthritis. Acta Orthop Scand 1969;40:346.

3 Hastings DE, Parker SM. Protrusio acetabuli in rheumatoid arthritis. Clin Orthop 1975;108:76.

4 Wilcock GK. The prevalence of osteoarthrosis of the hip requiring total hip replacement in the elderly. Int J Epidemiol 1979;8:247-50.

5 Gray RG, Gottlieb NL. Intra-articular corticosteroids - an updated assessment. Clin Orthop 1983;177:235-63.

6 Dieppe PA. Management of osteoarthritis of the hip and knee joints. Curr Opin Rheumatol 1993;5:487-93.

7 Ledingham J, Dawson S, Preston B, Milligan G, Doherty $M$. Radiographic patterns and associations of osteoarthritis of the hip. Ann Rheum Dis 1992;51:1111-6.

8 Ledingham J, Dawson S, Preston B, Milligan G, Doherty $M$. radiological progression of hopital referred osteoarthritis of the hip. Ann Rheum Dis 1993;52:263-7.

9 Menkes CJ, Decraemere W, Postel M, Forest M. Chondrocalcinosis and rapid destruction of the hip. J Rheumatol 1985;12:130-3.

10 Resnick D: Patterns of migration of the femoral head in osteoarthritis of the hip. Roentgenographic-pathologic correlation and comparison with rheumatoid arthritis. Am J Roentgenol 1975;124:62-74.

11 McGoldrick F, O'Brien T. Osteoarthritis of the hip and Heberden's nodes. Ann Rheum Dis 1989;48:53-5.

12 Kellgren JH, Lawrence JS. Radiological assessment of osteo-arthrosis. Ann Rheum Dis 1957;16:494-502.

13 Larsen A, Dale, K, Eek M. Radiographic evaluation of rheumatoid arthritis and related conditions by the rheumatoid arthritis and related conditions by the 91.

14 Solomon L. Patterns of osteoarthritis of the hip. J Bone Joint Surg Br 1976;58:176-83.

15 Thomas RH, Resnick D, Alazraki NP, Daniel D, Greenfield R. Compartmental evaluation of osteoarthritis of the knee. Radiology 1975;116:585-94.

16 Altman RD, Fries JF, Bloch DA, et al. Radiograhic assessment of progression in osteoarthritis. Arthritis Rheum 1987;30:1214-25.

17 Foldes K, Balint P, Gaal M, Buchanan WW, Balint GP. Nocturnal pain correlates with effusions in diseased hips. $J$ Rheumatol 1992;19:1756-8.

18 Flanagan J, Thomas TL, Casale FF, Desai KB. Intraarticular injection for pain relief in patients awaiting hip replacement. Ann Roy Coll Surg Engl 1988;70:156-7.

19 Leveaux VM, Quin CE. Local injections of hydrocortisone and procaine in osteoarthritis of the hip. Ann Rheum Dis 1956;15:330.

20 Smith RW, Cook PL, Cawley MID. A survey of arthrography and intra-articular corticosteroid injection of the hip joint. Br J Rheumatol 1994;33 (suppl 1):76.

21 Chakravarty K, Pharoah PDP, Scott DGI. A randomised controlled study of post-injection rest following intraarticular steroid therapy for knee synovitis. Br J Rheumatol 1994;33:464-8.

22 Gray RG, Tenenbaum J, Gottlieb NL. Local corticosteroid treatment in rheumatic disorders. Semin Arthritis Rheum 1981:10:231-54.

23 Gaffney K, Ledingham J, Perry JD. Intra-articular triamcinolone hexacetonide in knee osteoarthritis - factors influencing the clinical response. Ann Rheum Dis 1995; 54:379-81.

24 Jones $A$, Doherty $M$. Intra-articular corticosteroids are effective in osteoarthritis but there are no clinical predictors of response. Ann Rheum Dis 1996;55:829-32. 